
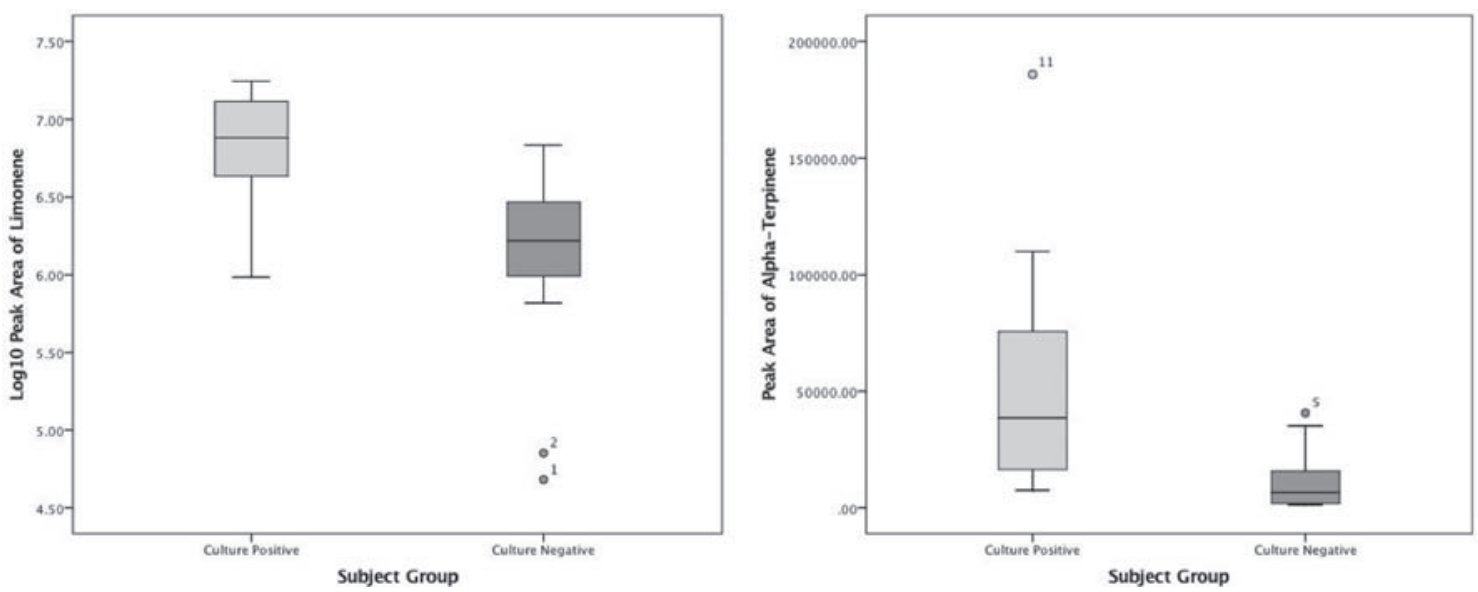

Abstract P111 Figure 1 Box-and-whisker plots of relative VOC abundance in culture-positive and culture-negative groups.

$(\mathrm{n}=11)$. Analysis of patient demographics and potential breath contaminants revealed no significant difference between groups. The targeted analysis incorporated 35 VOCs. Of these, 2 were found to be significantly more abundant (figure 1) in culture-positive than culture-negative patient samples. These compounds most likely represent limonene $(p=0.017)$ and alpha-terpinene $(p=0.007)$.

Conclusion This pilot study has identified differences in the exhaled VOC profile of patients with sputum culture-positive and culture-negative pulmonary aspergillosis. The finding of elevated levels of terpenoids in the breath of culture positive patients concurs with current understanding of the Aspergillus volatome in vivo and in vitro. A larger study using multivariate analysis should be performed to identify discriminating exhaled VOC signatures in patients with aspergillosis for future clinical use.

\section{P112 INCIDENCE AND CLINICAL OUTCOMES OF PULMONARY INFECTION WITH ACHROMOBACTER SPP}

${ }^{1}$ ZA Syed, ${ }^{2} \mathrm{~W}$ Flight. ${ }^{1}$ Christ Church College, University of Oxford, Oxford, UK; ${ }^{2}$ Oxford University Hospitals NHS Foundation Trust, Oxford, UK

\subsection{6/thoraxjn-2017-210983.254}

Background Achromobacter is considered an emerging Gramnegative pathogen in cystic fibrosis (CF) although its pathogenic potential is controversial and its impact in other patients groups largely unknown.

Methods All Achromobacter-positive respiratory samples analysed at our institution from 2007-2016 were identified. Antibiotic susceptibility was tested using the disc-diffusion method. Clinical data were collected from medical records. The Leeds criteria were adapted to define chronicity of infection.

Results 48 patients were identified, with a diagnosis of CF in 25 , bronchiectasis in 16 and miscellaneous other conditions in 7. Median (IQR) number of samples provided was 4 (1-16), $1.5(1-2.25)$ and $1(1-1.5)$ in the CF, bronchiectasis and other groups respectively. In the $\mathrm{CF}$ group, the median age at first Achromobacter isolate was 22.3 years (IQR 17-30) and mean BMI $21.8 \mathrm{~kg} / \mathrm{m}^{2}$ (SD 4.1). Baseline $\mathrm{FEV}_{1} \%$-predicted in the CF population was $50.4 \%$ (SD 22.6\%). Among bronchiectasis patients, median age at first isolate was 61 years (IQR 58-78)

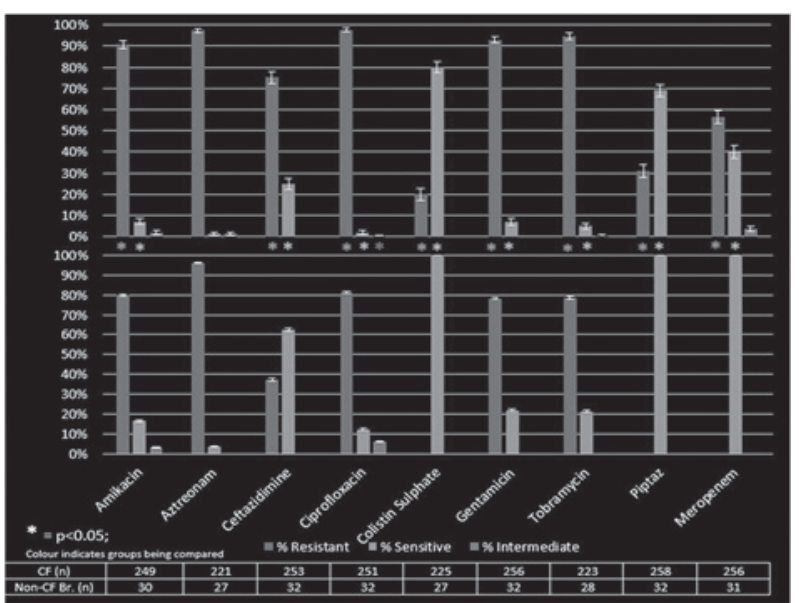

Abstract P112 Figure 1 Achromobacter spp. antibiotic sensitivities in CF (above) and non-CF Bronchiectasis (below) patients.

and $\mathrm{FEV}_{1} 1.35 \mathrm{~L} / \mathrm{s}$ (SD 0.75). During the study period, the yearly rate of detection of Achromobacter infection increased across all groups, with 4 cases in 2007 rising to 25 in 2016 and a mean of 9.9 cases/year. In any given year during the period 2012-2016, the mean proportion of CF patients with chronic, intermittent and cleared infection was 25\%, 54\% and $29 \%$ respectively, whilst in the bronchiectasis group it was 11\%, 37\% and 57\%. Co-infection with Staphylococcus aureus was seen in $20 \%$ of samples from CF patients. Equivalent figures for other pathogens were: Pseudomonas aeruginosa 14\%, Aspergillus 11\% and non-tuberculous mycobacteria $7 \%$. In the bronchiectasis group, co-infection was mainly seen with Pseudomonas aeruginosa (15\%). Antibiotic resistance was significantly greater in the CF group compared to the bronchiectasis group, particularly to meropenem $(57 \%$ of $\mathrm{CF}$ isolates vs $0 \%$ of bronchiectasis isolates), piperacillin-tazobactam (31\% vs $0 \%$ ) and colistin $(20 \%$ vs $0 \%) ; \mathrm{p}<0.05$.

Conclusions Detection of Achromobacter spp from respiratory samples increased over the study period with CF patients more likely to develop chronic infection. Achromobacter spp isolates from CF patients had greater levels of antibiotic resistance than those from patients with bronchiectasis. 